\title{
THREE-DIMENSIONAL FUZZY CONTROL OF ULTRASONIC CLEANING
}

\author{
Volodymyr MORKUN*(i) , Olha KRAVCHENKO*(i) \\ *Kryvyi Rih National University, 11, Vitaliy Matusevych Street, Kryvyi Rih 50027, Ukraine \\ morkunv@gmail.com, kravchenko@knu.edu.ua
}

received 3 September 2020, revised 12 July 2021, accepted 15 July 2021

\begin{abstract}
Consideration of ultrasonic cleaning as a process with distributed parameters enables reduction of power consumption. This approach is based on establishment of control over the process depending on fixed values of ultrasonic responses in set points. The initial intensity of radiators is determined using a three-dimensional (3D) interval type-2 fuzzy logic controller essentially created for processes with distributed parameters, as well as complex expert evaluation of the input data. The interval membership functions for the input and output data consider the space heterogeneity of ultrasonic cleaning. A rule base is formed, which is $2 \mathrm{D}$ and not dependent upon the number of input and output parameters. A model illustrating ultrasonic cleaning with a 3D interval type-2 fuzzy logic controller is designed. Comparative analysis of the output parameters of the proposed model and the traditional method indicates an increase in the energy efficiency by $41.17 \%$ due to application of only those ultrasonic radiators that are located next to the contamination.
\end{abstract}

Keywords: ultrasonic cleaning, 3D fuzzy interval type-2 logic, modeling, spatially distributed systems

\section{INTRODUCTION}

Effective cleaning guarantees both long-term exploitation of equipment and possibility of current maintenance. Ultrasonic cleaning is noted for higher efficiency and sustainability as it reduces the application of chemical solvents. Ultrasonic cleaning is based on the cavitation effect - implosion of bubbles resulting from the ultrasonic processing of a liquid. Consequently, energy that is capable of separating particles of dirt from the cleaned object is emitted. Issues related to enhancing the efficiency of ultrasonic cleaning are considered from two perspectives: as an investigation into parameters affecting a process in order to improve cavitation activity; and as establishment of control considering the peculiarities of ultrasonic cleaning. Cavitation intensity is not the same in different zones of an ultrasonic bath and depends on a number of factors. Space configuration of an ultrasonic bath, i.e., the size of the container, as well as the number and location of ultrasonic radiators, plays a major part in the process (Tangsopha and Thongsri, 2020). The frequency of insonation also affects cavitation distribution in space (Tangsopha et al., 2017). Temperature, intensity of processing, and oxygen content also influence the process, yet they only correct the intensity of cavitation in the ultrasonic bath without changing its distribution. While studying the parameters affecting cavitation, Xu et al. (2016) report the following dependencies: cavitation becomes unstable with long intonation, which is more vivid with greater capacity; increased gas content, capacity, and addition of a cleaning agent enhance cavitation, but on reaching an extreme value of these parameters, the cavitation activity falls. With relatively great capacity, cavitation increases with temperature, reaches its peak, and then drops. Besides affecting cavitation, these parameters are also interrelated: duration of insonation and high capacity influence temperature and gas content, while the latter depends on temperature. In other words, this is a complex physical process with a great number of interrelated impact factors, the values of which change greatly at different points of a cleaning container.

Nigmetzyanov et al. (2019) present an attempt to consider the space distribution of cavitation by using an additional lowamplitude radiator to increase the efficiency of cavitation collapses in a remote zone of cavitation activity. Yet, this issue of automatizing cleaning considering the space distribution remains unsolved. Available systems of controlling ultrasonic cleaning are constrained either by time and expert evaluation regarding the need to continue the process or by assessment of the condition of the cleaning liquid including temperature, conductivity, and turbidity (Duran and Teke, 2018; Rahim et al., 2011). This leads to the fact that processing time - and consequently, power consumption - is not associated with the real contamination of a body. In other words, there arises a situation when insonation is related to the cleaned areas of a product. To avoid this, one should use an algorithm controlling ultrasonic cleaning, which will consider contamination of a body in terms of the body's separate areas. Assessing this condition through analysis of ultrasonic reflections in the bath is suggested because ultrasonic measurements provide high-quality and fast evaluation of space-distributed parameters (Porkuian et al., 2019, 2020; Morkun et al., 2015a). A threedimensional (3D) interval type-2 (IT2) fuzzy logic controller, which is essentially created to control systems with distributed nonlinear parameters, is applied to the process of determining the establishment of a distributed controlling action (Li et al., 2007; Zhang et al., 2017).

\section{PROBLEM DESCRIPTION}

Let there be a system with distributed parameters describing the process of ultrasonic cleaning. Cleaning occurs due to $m$ ultrasonic radiators located in set positions and set by intensity factors $u(z)=\left(u\left(z_{1}\right), u\left(z_{2}\right), \ldots, u\left(z_{m}\right)\right)$. The input parame- 
ters are the measures in $P$ space positions $Z_{1}, Z_{2}, \ldots Z$. Moreover, $P$ and $m$ are independent. Figure 1 depicts a bath for ultrasonic cleaning. Here, $\Delta x_{z}=\left(\Delta x_{z_{1}}, \Delta x_{z_{2}}, \ldots, \Delta x_{z_{p}}\right)$ goes to the system input and is the difference between the two latest measurements of the signal $\Delta x_{z}=\left|x_{z}{ }^{(1)}-x_{z}\right|$ and $x_{z}=$ $\left(x_{z_{1}}, x_{z_{2}}, \ldots, x_{z_{p}}\right)$, the value of the latest measurement. The intensity of the radiators is determined by the controller while analyzing the input parameters. The input parameters are measured by ultrasonic sensors that fix the reflection from the cleaned body in the set time intervals. It is necessary to establish control in such a way that cleaning is performed with minimum energy consumption considering the space distribution of dirt on the object. The space position is set by a $1 \mathrm{D}$ radial coordinate.

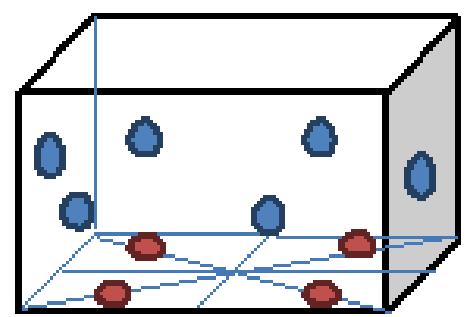

Fig. 1. Diagram of the ultrasonic cleaning bath with the sensors on its sides and the basic cleaning radiators on its bottom
To establish control of the set, a 3D IT2 fuzzy logic controller is used; its functioning algorithm is described in Section 3. Simulation of the controller functioning and comparison of its efficiency with the conventional method of cleaning is performed.

\section{DEPENDENCY OF ULTRASONIC REFLECTIONS AND DEVELOPMENT OF CLEANING}

Modeling ultrasonic cleaning (Figs. 2 and 3) applying k-wave (Treeby and Cox, 2010) reveals the dependency of the cleaning process and the ultrasonic reflections of a situational character. In other words, a signal can be affected by both dirt peeling, as seen in Fig. 2 (increased amplitude and reduced number of dispersed deviations), and the geometry of the cleaned sections (in Fig. 3, cleaning of the hole increases the dispersed deviations). For this reason, the following assumptions are used to determine the running of the process:

1. The process occurs as cleaning only, i.e., any change of a sensor signal indicates dirt peeling.

2. The sensor closest to the dirt peeling records the greatest change of a signal.
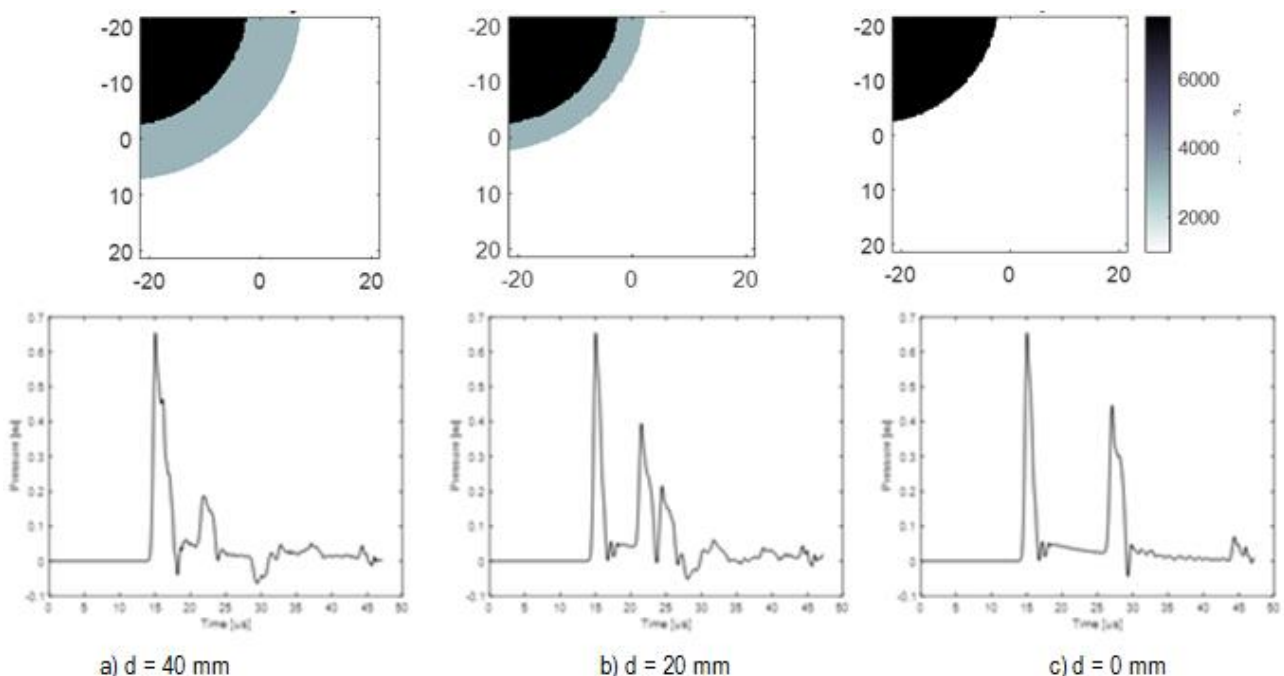

b) $\mathrm{d}=20 \mathrm{~mm}$

c) $\mathrm{d}=0 \mathrm{~mm}$

Fig. 2. Acoustic pressure during the processing of a body with contamination density $\rho=3,100 \mathrm{~kg} / \mathrm{m}^{3}$, velocity of ultrasonic waves $c=2,500 \mathrm{~m} / \mathrm{sec}$, and contamination thickness $d$ (the cleaned body has $\rho=7,800 \mathrm{~kg} / \mathrm{m}^{3}$ and the ultrasonic wave velocity $c=5,170 \mathrm{~m} / \mathrm{sec}$ ) for sector of the circle

\section{CONTROLLING ULTRASONIC CLEANING BY 3D IT2 FUZZY LOGIC CONTROLLER}

Modern industrial advance calls for higher standards of energy efficiency and safety. Conventional methods of controlling systems with concentrated parameters cannot meet these standards, thus causing the necessity to use methods considering the space distribution of the system (Morkun et al., 2015b). The high complexity of mathematical models of these systems almost eliminates their practical application (Morkun and Tron, 2014a; Morkun et al., 2015c). To avoid complex and bulky mathematical models, training algorithms and expert experience are used (Morkun et al., 2014b, 2015d). A 3D fuzzy logic controller is essentially created to control systems with distributed parameters (Li et al., 2007; Zhang et al., 2017). Expansion of the traditional fuzzy set by an additional space coordinate enables the processing of space data. When applying this controller to automatizing ultrasonic cleaning, space distribution of the system is used. The process is assessed by the changes and the "purity" of the ultrasonic responses in the cleaning container. Expert assessment of this parameter is quite variable. This problem is solved by applying an interval membership function enabling usage of expert assessment in the form of a range.

Thus, a combination of a 3D fuzzy logic controller ( $\mathrm{Li}$ et al., 2007) and an IT2 fuzzy logic controller (Zadeh, 1975) serves as 
the basis for a 3D interval fuzzy logic controller. It enables the establishment of control over ultrasonic cleaning with due regard for its space distribution in the absence of clear expert assessment of the significance of changes in the ultrasonic response.
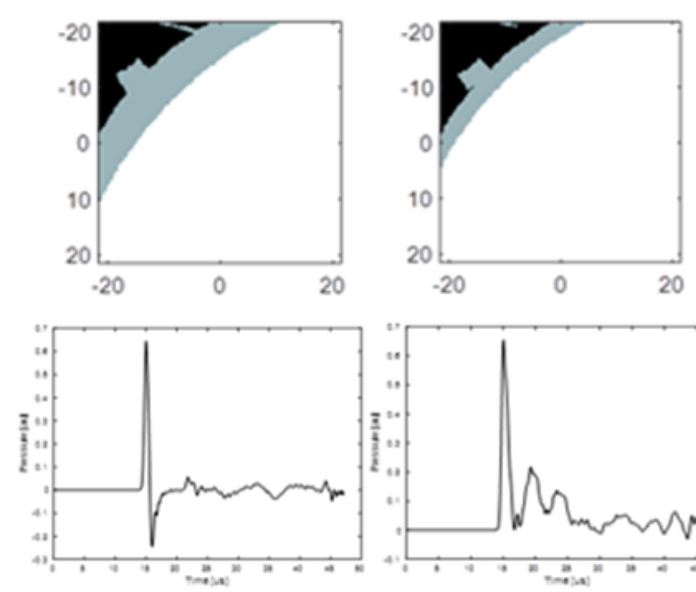

a) $\mathrm{d}=41 \mathrm{~mm}$

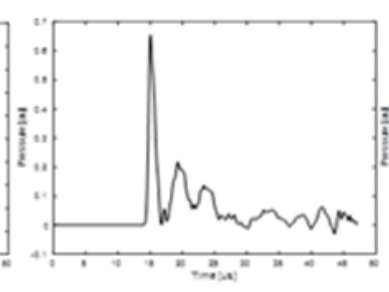

b) $\mathrm{d}=30 \mathrm{~mm}$
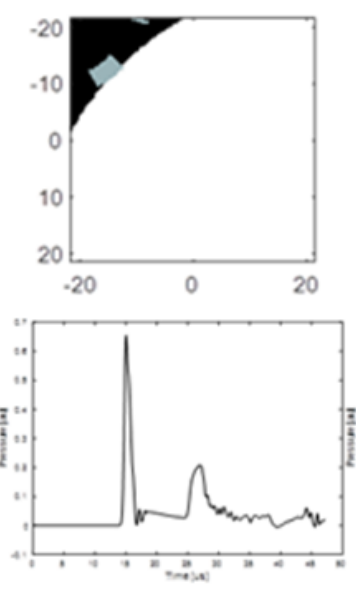

c) contaminated hole only
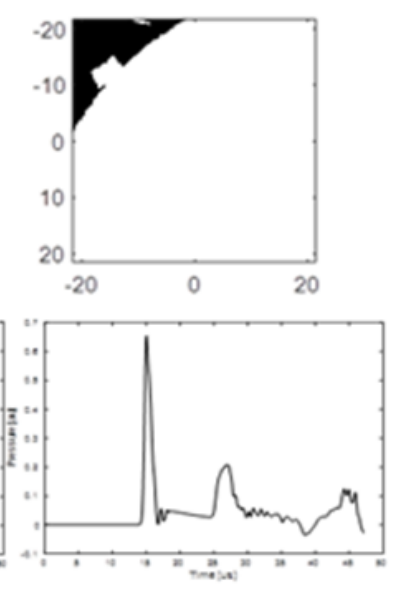

d) clean body

Fig. 3. Acoustic pressure during the processing of a body with contamination density $\rho=3,100 \mathrm{~kg} / \mathrm{m}^{3}$, ultrasonic wave velocity $c=2,500 \mathrm{~m} / \mathrm{sec}$, and contamination thickness $d$ (the cleaned body has $\rho=7,800 \mathrm{~kg} / \mathrm{m}^{3}$ and the ultrasonic wave velocity $c=5,170 \mathrm{~m} / \mathrm{sec}$ ) for sector of the astroid

The membership function and the rule base

According to the problem description, the crisp inputs are $x_{1}(z)$ and $x_{2}(z)$, where $z=\left(z_{1}, z_{2}, . . z_{p}\right)$, denoting the changed ultrasonic responses in the latest cleaning cycle and the values of the latest indices of sensors, respectively. The crisp output indicates the intensity of functioning of the ultrasonic radiators in set positions $u(z)=\left[u\left(z_{1}\right), u\left(z_{2}\right), \ldots u\left(z_{m}\right)\right]$. The values of $x_{1}(z), x_{2}(z)$ and $u(z)$ are normalized within $[0,1]$. All the variables have three linguistic marks: large $(\mathrm{L})$, medium $(\mathrm{M})$, and small $(\mathrm{S})$. Considering the space distribution of ultrasonic cleaning, the upper and the lower membership functions of the input parameters appear as follows:

$\underline{\mu}\left(x_{i}, z\right)=\exp \left(-\frac{\left(\left(x_{i}-a\right)^{2}+\left(|2| z-z_{0}|-1|-1\right)^{2}\right)}{\sigma_{1}}\right)$

$\bar{\mu}\left(x_{i}, z\right)=\exp \left(-\frac{\left(\left(x_{i}-a\right)^{2}+\left(|2| z-z_{0}|-1|-1\right)^{2}\right)}{\sigma_{2}}\right)$

$i=1,2, z=\left(z_{1}, z_{2}, \ldots, z_{P}\right)$

where $\underline{\mu}\left(x_{i}, z\right)$ and $\bar{\mu}\left(x_{i}, z\right)$ are the upper and lower values of the membership function, respectively; $x_{i}$ is the crisp input; $z$ is the radial input coordinate; $a$ is the value of the linguistic mark with which the membership function acquires the maximum value: for large (L): $a=1$; for medium (M): $a=0.5$; and for small (S): $a$ $=0, z_{0}$ is the value of the spacious radial output coordinate, $\sigma_{1}$ and $\sigma_{2}$ are the parameters conditioned by the physical properties of both the cleaning container as well as the number of sensors and outputs and belong to the interval $[0,1]$; and $i$ is the number of variables. Figure 4 shows a diagram for the medium linguistic mark (M) and the output $z_{0}=0.2$ and the small mark $(\mathrm{S})$ and the output $z_{0}=0.5$ with $\sigma_{1}=0.1$ and $\sigma_{2}=0.2$. The graph indicates the maximum value in the space point closest to the output, and this corresponds to the linguistic mark.

For the output $u(z)$, the membership function is defined in the trapezoid-triangular form, as shown in Fig. 5. The membership function is $2 \mathrm{D}$ as the spatial impact is taken into account during the fuzzification of the input, while the output value is calculated depending on its space location.

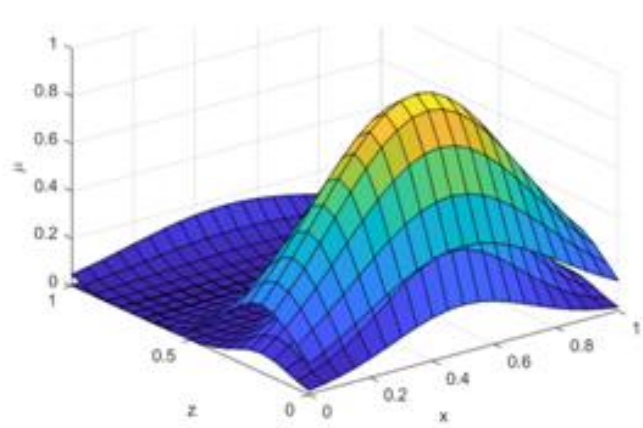

a)

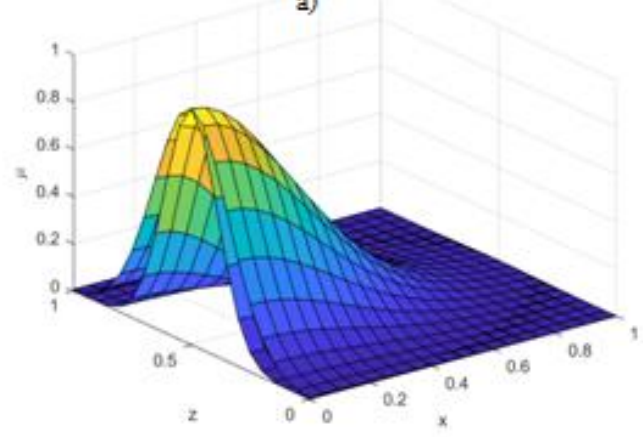

b)

Fig. 4. Diagrams of the upper and lower membership functions: a) for the medium mark $(M)$, for the output $z_{0}=0.2$; b) for the small mark (S), for the output $z_{0}=0.5$

The rule base (Table 1) considers the assumptions presented above: as change of a signal $\Delta x_{z}$ is the major evidence of successful cleaning, it is considered more significant than the latest signal $x_{z}$ conditioned not only by the contamination degree of a product, but also by its geometry and location in the container.

On the basis of the determined membership functions and the rule base, we describe the algorithm of the controller functioning based on the 3D IT2 fuzzy logic set (FS). 


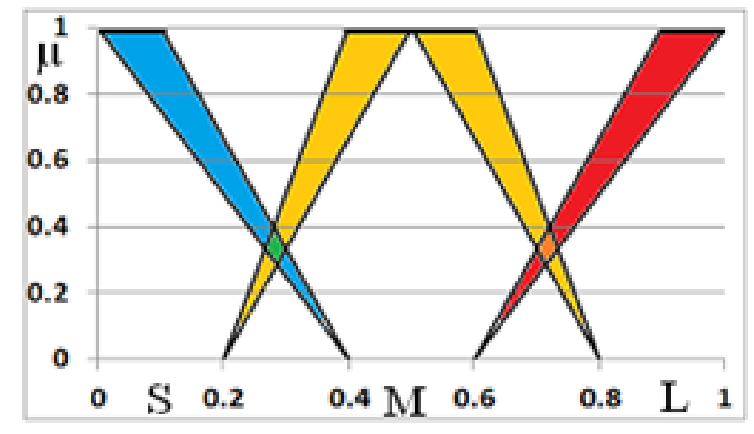

Fig. 5. Membership function for output $u(z)$. S - small, M-medium, Llarge.

Tab. 1. Rule base ( $\tilde{L}$ - large, $\tilde{M}$ - medium, and $\tilde{S}$ - small)

\begin{tabular}{|c|c|c|c|}
\hline$\Delta \boldsymbol{x}_{\boldsymbol{z}} / \boldsymbol{x}_{\boldsymbol{z}}$ & $\tilde{\boldsymbol{S}}$ & $\widetilde{\boldsymbol{M}}$ & $\tilde{\boldsymbol{L}}$ \\
\hline$\tilde{\boldsymbol{S}}$ & $\widetilde{M}$ & $\tilde{S}$ & $\tilde{S}$ \\
\hline$\widetilde{\boldsymbol{M}}$ & $\widetilde{M}$ & $\widetilde{M}$ & $\tilde{S}$ \\
\hline$\tilde{\boldsymbol{L}}$ & $\tilde{L}$ & $\tilde{L}$ & $\widetilde{M}$ \\
\hline
\end{tabular}

\section{THE 3D IT2 FS CONTROLLER}

The concept of a 3D fuzzy set is introduced to enable consideration of the space distribution of complex physical and chemical processes (Li et al., 2007; Zhang et al., 2017). The controller based on this set is naturally created for processes with distributed parameters and can be applied to the controlling of ultrasonic cleaning. Expansion of the membership function in the given controller to reach the first interval results in changes of the input formation mechanism. Let us consider the operations within the
3D IT2 FS controller structure.

The structure of the 3D IT2 FS controller is similar to that of the basic one (Mamdani, 1974) with standard operations of fuzzification, the fuzzy logic output, and defuzzification (Fig. 6). Yet, availability of an additional space coordinate and intervals of the membership function call for their expansion. We provide all the controller operations for singleton fuzzification to simplify and contract them.

For the 3D fuzzy set, we determine the fuzzification of the crisp input $X$ in the following way (Li et al., 2007):

$$
\begin{aligned}
& \tilde{A}_{x_{1}}=\sum_{z \in Z} \sum_{x_{1}(z) \in X_{1}} \frac{\mu_{x_{1}}\left(x_{1}(z), z\right)}{\left(x_{1}(z), z\right)}, \text { where } \mu_{x_{1}}\left(x_{1}(z), z\right)= \\
& \int_{u \in J_{x_{1}}}\left[\underline{\mu}_{\tilde{A}}\left(x_{1}(z), z\right), \bar{\mu}_{\tilde{A}}\left(x_{1}(z), z\right)\right] \frac{1}{u}
\end{aligned}
$$

$\tilde{A}_{x_{N}}=$

$\sum_{z \in Z} \sum_{x_{N}(z) \in X_{N}} \frac{\mu_{x_{N}}\left(x_{N}(z), z\right)}{\left(x_{N}(z), z\right)}$, where $\mu_{x_{N}}\left(x_{N}(z), z\right)=$ $\int_{u \in J_{x_{N}}}\left[\underline{\mu}_{\tilde{A}}\left(x_{N}(z), z\right), \bar{\mu}_{\tilde{A}}\left(x_{N}(z), z\right)\right] \frac{1}{u}$,

where $u$ is the secondary variable, which is an interval for the membership function with the sets $x$ and $z ; J_{x_{1}}, \ldots, J_{x_{N}}$ determine the interval of the membership function for the set $x_{1}, \ldots, x_{N}$. In the case of ultrasonic cleaning, $\underline{\mu}_{\tilde{A}}\left(x_{i}(z), z\right), \bar{\mu}_{\tilde{A}}\left(x_{i}(z), z\right)$ correspond to $\mu\left(x_{i}, z\right)$ and $\bar{\mu}\left(x_{i}, z\right)$ from Eq. (1), while $N=2$. Thus, the general fuzzification for two crisp inputs looks as follows:

$\tilde{A}_{x=} \sum_{z \in Z} \sum_{x_{1}(z) \in X_{1}} \sum_{x_{2}(z) \in X_{2}} \frac{\mu_{x_{1}}\left(x_{1}(z), z\right) * \mu_{x_{1}}\left(x_{1}(z), z\right)}{\left(x_{1}(z), x_{2}(z), z\right)}$,

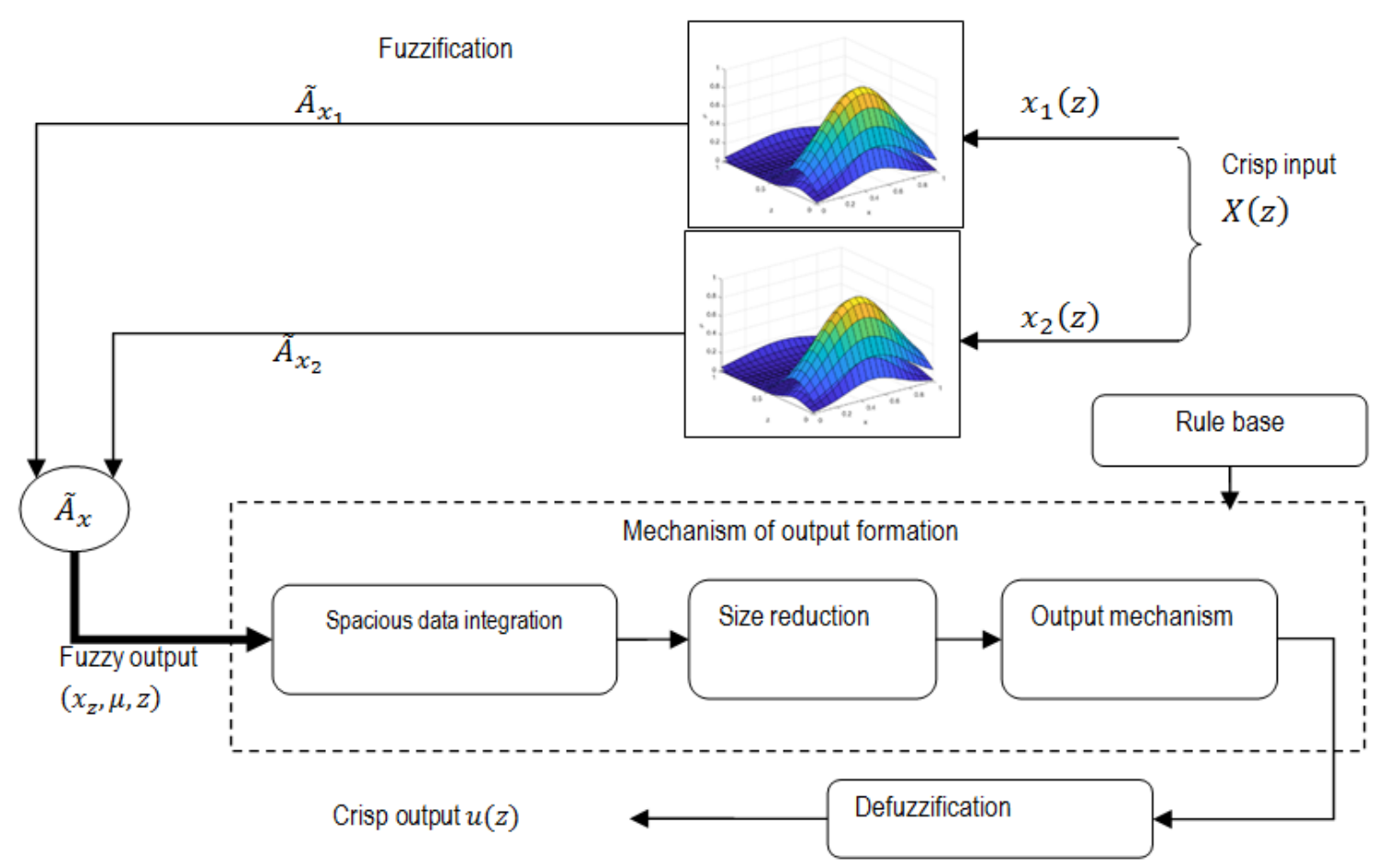

Fig. 6. The 3D IT2 FS controller 
To establish control over ultrasonic cleaning, we define a t-norm as an intersection of sets, i.e., for $\tilde{A}_{x_{1}}$ and $\tilde{A}_{x_{2}}$, we have:

$$
\begin{aligned}
& \tilde{A}_{x_{1}}=\frac{1}{U_{\forall x \in X, Z \in Z}\left[\underline{\mu}_{\widetilde{A}_{x_{1}}}(x, z), \bar{\mu}_{\widetilde{A}_{x_{1}}}(x, z)\right]} ; \widetilde{A}_{x_{2}}= \\
& \frac{1}{U_{\forall x \in X, z \in Z}\left[\underline{\mu}_{\widetilde{A}_{x_{2}}}(x, z), \bar{\mu}_{\widetilde{A}_{x_{2}}}(x, z)\right]} \\
& \frac{\tilde{A}_{x_{1}} \cap \tilde{A}_{x_{2}}=}{U_{\forall x \in X, z \in Z}\left[\underline{\mu}_{\widetilde{A}_{x_{1}}}(x, z) \wedge \underline{\mu}_{\widetilde{A}_{x_{2}}}(x, z), \bar{\mu}_{\widetilde{A}_{x_{1}}}(x, z) \wedge \bar{\mu}_{\widetilde{A}_{x_{2}}}(x, z)\right]}= \\
& \frac{1}{U_{\forall x \in X, z \in Z}\left[\min \left(\underline{\mu}_{\widetilde{A}_{x_{1}}}(x, z), \underline{\mu}_{\widetilde{A}_{x_{2}}}(x, z)\right), \min \left(\bar{\mu}_{\widetilde{A}_{x_{1}}}(x, z) \wedge \bar{\mu}_{\widetilde{A}_{x_{2}}}(x, z)\right)\right]}
\end{aligned}
$$

After fuzzification of the crisp inputs, the fuzzy logic output is established on the basis of the set rule base. According to Table 1 , the 1 -th rule in the base can be written in the following way:

$\widetilde{R}^{l}$ : If $x_{1}(z)$ is $\widetilde{C}_{1}^{l}$ and $x_{2}(z)$ is $\widetilde{C}_{2}^{l}$ then $u(z)$ is $\widetilde{K}^{l}$

where $\tilde{R}^{l}$ is the $l$-th rule $(I=1,2 \ldots 9) ; x_{1}(z)$ and $x_{2}(z)$ are the input variables in different points of the space; $\tilde{C}_{1}^{l}$ and $\tilde{C}_{2}^{l}$ are the 3D IT2 FSs, and $u(z)$ is the controlling action. The next step is to reduce the 3D IT2 FS to conventional IT2 FS. With this, integration of spacious data, size reduction, and output establishment occur. Rule (5) can be written as the following fuzzy ratio:

$\tilde{R}^{l}: \tilde{A}_{1}^{l} \times \tilde{A}_{1}^{l} \rightarrow \widetilde{K}^{l}, l=1,2, \ldots 9$

The IT2 FS is obtained as a combination of the 3D IT2 FS at the input and application of the rule in the form of fuzzy ratios. Integration of space data results in transformation of the 3D fuzzy logic interval input $\tilde{A}_{x}$ into the $3 \mathrm{D}$ set $W$, which is an IT2 FS in each point $x_{z}=\left[x_{1}(z), x_{2}(z)\right]$. The set $W$ is an expanded composition of input sets. Figure 7 shows this composition for the singleton value. The expanded composition performed at the input set and the assigned sets of the I-th rule are presented by Li et al. (2007):

$W_{\tilde{A}_{x^{\circ}}\left(\tilde{C}_{1}^{l} \times \tilde{C}_{2}^{l}\right)}^{l}=\widetilde{A}_{x} \circ\left(\tilde{C}_{1}^{l} \times \tilde{C}_{2}^{l}\right)$

with the upper and the lower values of the membership function:

$\underline{\mu}_{W^{l}}=\underline{\mu}_{\tilde{A}_{x^{\circ}}\left(\tilde{C}_{1}^{l} \times \tilde{C}_{2}^{l}\right)}\left(x_{z}, z\right)=$

$\sup _{x_{1}(z) \in X_{1}, x_{2}(z) \in X_{2}}\left[\underline{\mu}_{\tilde{A}_{x}}\left(x_{z}, z\right) * \underline{\mu}_{\tilde{C}_{1}^{l} \times \tilde{C}_{2}^{l}}\left(x_{z}, z\right)\right]=$

$\sup _{x_{1}(z) \in X_{1}, x_{2}(z) \in X_{2}}\left[\underline{\mu}_{x_{1}}\left(x_{1}(z), z\right) * \underline{\mu}_{x_{2}}\left(x_{2}(z), z\right) *\right.$

$\left.\underline{\mu}_{\tilde{C}_{1}^{l}}\left(x_{1}(z), z\right) * \underline{\mu}_{\tilde{C}_{2}^{l}}\left(x_{2}(z), z\right)\right]=$

$\left\{\sup _{x_{1}(z) \in X_{1}}\left[\underline{\mu}_{x_{1}}\left(x_{1}(z), z\right) * \underline{\mu}_{\tilde{C}_{1}^{l}}\left(x_{1}(z), z\right)\right]\right\} *$

$\left\{\sup _{x_{2}(z) \in X_{2}}\left[\underline{\mu}_{x_{2}}\left(x_{2}(z), z\right) * \underline{\mu}_{\tilde{C}_{2}^{l}}\left(x_{2}(z), z\right)\right]\right\}$

$\bar{\mu}_{W^{l}}=\bar{\mu}_{\tilde{A}_{x^{\circ}}\left(\tilde{C}_{1}^{l} \times \tilde{C}_{2}^{l}\right)}\left(x_{z}, z\right)=$

$\sup _{x_{1}(z) \in X_{1}, x_{2}(z) \in X_{2}}\left[\bar{\mu}_{\tilde{A}_{x}}\left(x_{z}, z\right) * \bar{\mu}_{\tilde{C}_{1}^{l} \times \tilde{C}_{2}^{l}}\left(x_{z}, z\right)\right]=$

$\sup _{x_{1}(z) \in X_{1}, x_{2}(z) \in X_{2}}\left[\bar{\mu}_{x_{1}}\left(x_{1}(z), z\right) * \bar{\mu}_{x_{2}}\left(x_{2}(z), z\right) *\right.$

$\left.\bar{\mu}_{\tilde{C}_{1}^{l}}\left(x_{1}(z), z\right) * \bar{\mu}_{\tilde{C}_{2}^{l}}\left(x_{2}(z), z\right)\right]=$

$\left\{\sup _{x_{1}(z) \in X_{1}}\left[\bar{\mu}_{x_{1}}\left(x_{1}(z), z\right) * \bar{\mu}_{\tilde{c}_{1}^{l}}\left(x_{1}(z), z\right)\right]\right\} *$

$\left\{\sup _{x_{2}(z) \in X_{2}}\left[\bar{\mu}_{x_{2}}\left(x_{2}(z), z\right) * \bar{\mu}_{\tilde{C}_{2}^{l}}\left(x_{2}(z), z\right)\right]\right\}$

where $z \in Z$, the product * indicates the t-norm operation (intersection of sets).

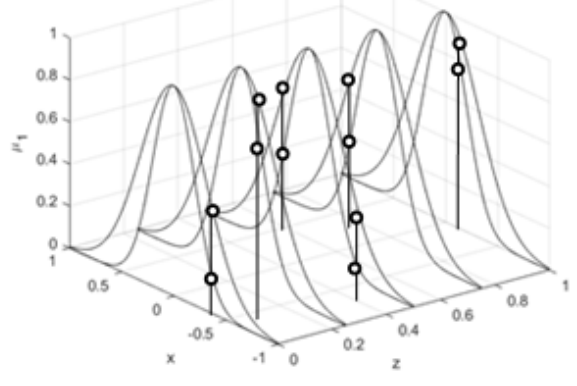

3-D IT2 FS

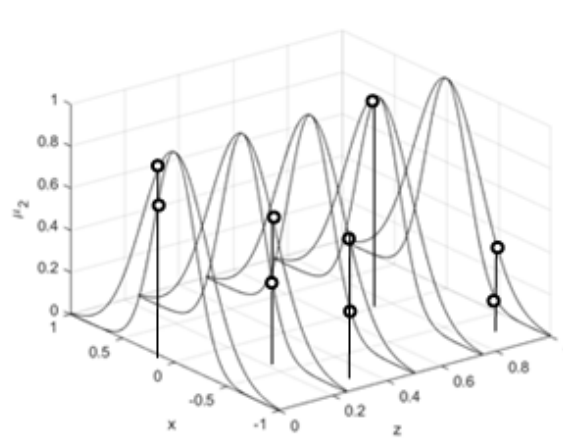

Fuzzification

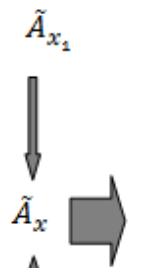

W

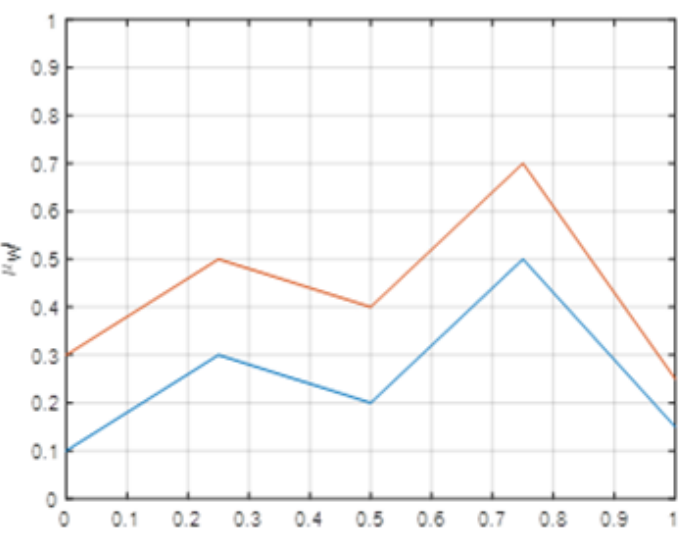

2-D interval type 2 space fuzzy logic membership function for

$$
x_{z}
$$

Integration

is performed by finding the maximum value, while the upper and the lower membership functions are determined as follows: 
Contamination peeling is recorded when the acoustic pressure is $>50 \%$ compared to the maximum. Experiments reveal that
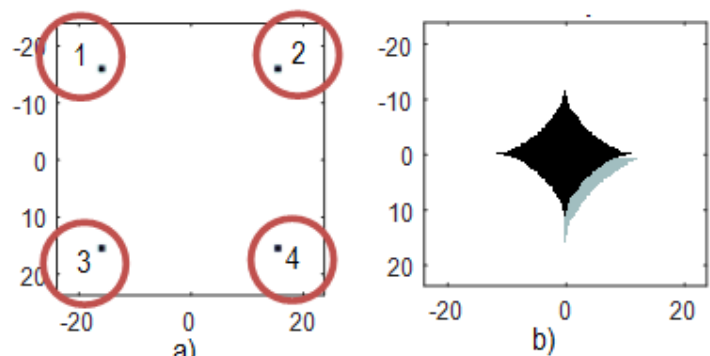

a) where $N^{\prime}$ is the number of production rules.

In compliance with the paper by Mendel et al. (2006), typecasting is performed by searching for the centroid of all production rules. It results in a collection of numbers with the maximum and the minimum values, i.e.:

$\mathrm{C}_{\tilde{B}}(z)=\frac{1}{\left\{c_{l}(z), \ldots, c_{r}(z)\right\}^{\prime}}$

where

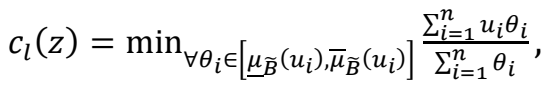

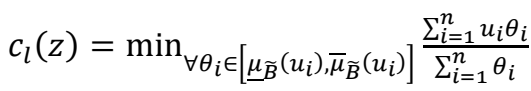

To find $c_{l}$ and $c_{r}$, we use the formulae of the Karnik-Mendel iteration algorithm (Karnik \& Mendel, 2001):

$c_{l}(z)=\frac{\sum_{i=1}^{l} u_{i}(z) \bar{\mu}_{\widetilde{B}}\left(u_{i}\right)+\sum_{i=l+1}^{N} u_{i}(z) \underline{\mu_{\widetilde{B}}}\left(u_{i}\right)}{\sum_{i=1}^{l} \bar{\mu}_{\widetilde{B}}\left(u_{i}\right)+\sum_{i=l+1}^{N} \underline{\mu}_{\widetilde{B}}\left(u_{i}\right)}$,

$c_{r}(z)=\frac{\sum_{i=1}^{r} u_{i}(z) \underline{\mu}_{\widetilde{B}}\left(u_{i}\right)+\sum_{i=r+1}^{N} u_{i}(z) \bar{\mu}_{\widetilde{B}}\left(u_{i}\right)}{\sum_{i=1}^{r} \underline{\mu}_{\widetilde{B}}\left(u_{i}\right)+\sum_{r=l+1}^{N} \bar{\mu}_{\widetilde{B}}\left(u_{i}\right)}$

Points $r$ and $l$ are determined iteratively by the Karnik-Mendel algorithm.

Defuzzification is reduced to a simple search for the mean value of the maximum and the minimum values after typecasting (Mendel et al., 2006):

$u=\frac{c_{r}+c_{l}}{2}$.

\section{MODELING CONTROL BY THE 3D IT2 FS CONTROLLER}

Ultrasonic cleaning is modeled by creating the ultrasonic pressure field with the assumption that the best cleaning occurs in zones with the largest values of acoustic pressure. Practical expediency of this assumption is confirmed by numerous experiments (Tangsopha et al., 2017; Tangsopha \& Thongsri, 2020; Nigmetzyanov et al., 2019). The $k$-wave software device is applied to modeling, enabling the simulation of large-scale ultrasonic waves in the set period (Treeby \& Cox, 2010; Treeby, et al., 2012; Morkun, et al., 2014).

As the space coordinate is actually single, we consider cleaning in a plane, i.e., sensors and radiators are in a single plane.

First, modeling without fuzzy logic control is performed. Four radiators of stable capacity are determined (Fig. 8a). The astroid with a single contaminated side is used as a cleaned product (Fig. 8b).

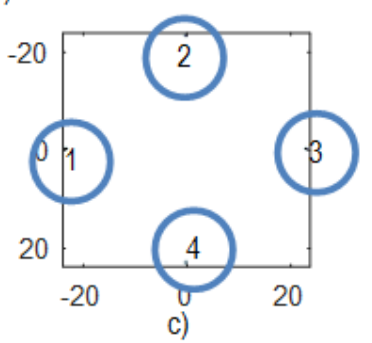

Fig. 8. Initial conditions of the experiment: a) location of the radiators; b) the view of the cleaned body; c) location of the sensors

complete cleaning covers five cycles. With that, all the radiators are engaged, and this stage corresponds to conventional ultrasonic cleaning.

While using fuzzy control, the initial conditions of the experiment remain the same. To assess the running of ultrasonic cleaning, four sensors are used, which are arranged as shown in Fig. $8 \mathrm{c}$. The sensors are both ultrasound radiators and receivers. Changes of signals are assessed by the difference between the amplitudes of the latest signals, while the number of changes of the signal sign becomes the basis for assessing the "purity" of the signal. Initiation occurs as a result of the response after the initial ultrasonic radiation by the sensors. The sensors are set by space positions $z=\left[\begin{array}{llll}0 & 0.25 & 0.5 & 0.75\end{array}\right]$, while the coordinates of the radiators are $z_{0}=\left[\begin{array}{lll}0.125 & 0.3750 .6250 .875\end{array}\right]$.

In the membership function (1), the following set values of the parameters $\sigma_{1}=0.1$ and $\sigma_{2}=0.2$ are used. After the initial processing according to the analysis of the maximum and the minimum values in each contamination point, the view of the cleaned object is obtained (Fig. 9).

The next step includes processing the body from all four basic radiators, followed up by ultrasonic assessment of the object's condition.

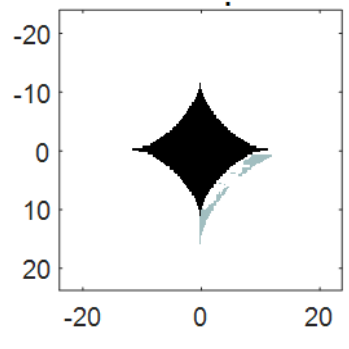

a)

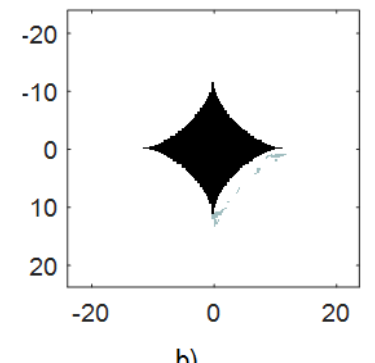

b)
Fig. 9. View of the body after $a$ ) the fist and b) the second cleaning cycles 
Tab. 2. Input $(\Delta x, x)$ and output (u) parameters in ultrasonic cleaning

\begin{tabular}{|c|c|c|c|c|}
\hline $\begin{array}{c}\text { Step } \\
\text { number }\end{array}$ & $\Delta x$ & $x$ & $u$ & $\begin{array}{c}\text { Power } \\
\text { saving, } \%\end{array}$ \\
\hline 0 & - & - & {$\left[\begin{array}{llll}1 & 1 & 1 & 1\end{array}\right]$} & 0 \\
\hline 1 & $\begin{array}{c}{[0.2292} \\
0.05981 .0000 \\
0.4948]\end{array}$ & $\begin{array}{c}{\left[\begin{array}{l}1.0000 \\
0.9167 \\
0.6667\end{array}\right]}\end{array}$ & 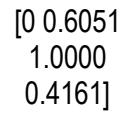 & 49.47 \\
\hline 2 & $\begin{array}{c}0.09180 .0026 \\
1.0000 \\
0.5896]\end{array}$ & $\begin{array}{c}{[1.0000} \\
1.0000 \\
0.50000]\end{array}$ & $\begin{array}{c}{\left[\begin{array}{ll}0 & 0.6253 \\
1.0000 \\
0.3537]\end{array}\right]} \\
\end{array}$ & 50.53 \\
\hline 3 & 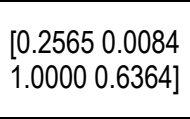 & $\begin{array}{c}{[1.0000} \\
1.0000 \\
0.36360]\end{array}$ & $\begin{array}{c}{\left[\begin{array}{ll}0 & 0.5488 \\
1.0000 \\
0.4900\end{array}\right]} \\
\end{array}$ & 49.03 \\
\hline 4 & 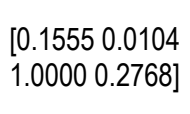 & 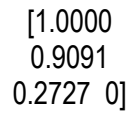 & $\begin{array}{c}{\left[\begin{array}{ll}0 & 0.5159 \\
1.0000 \\
0.2104]\end{array}\right]} \\
\end{array}$ & 56.84 \\
\hline Total & {$\left[\begin{array}{llll}0 & 0 & 0 & 0\end{array}\right]$} & & & 41.17 \\
\hline
\end{tabular}

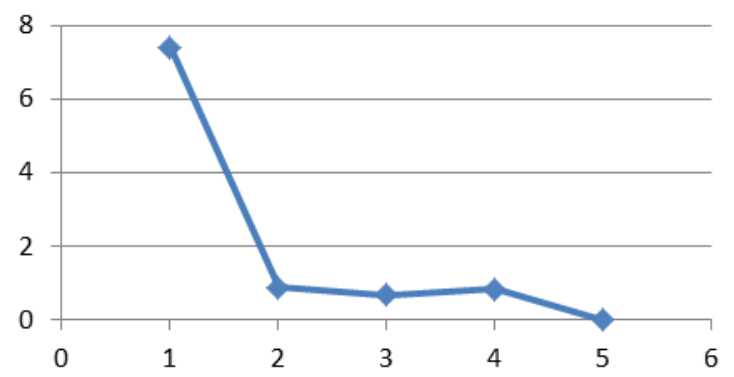

Fig.10. Reduction in the deviation vector module by the cleaning cycles

The normalized indices of the sensors obtained at Step 1 (Table 2) - change of the signal $\Delta x=\left[\begin{array}{lllll}0.2292 & 0.0598 & 1.0000 & 0.4948\end{array}\right]$ and the values of the latest measurements characterizing the degree of "purity" of the signal $x=\left[\begin{array}{llll}1.0000 & 0.9167 & 0.6667 & 0\end{array}\right]$ - are in compliance with both the assumptions about the greatest change of the signal of the sensor closest to the contamination peeling and a great number of deviations in the sensors in close proximity.

After analyzing the sensor data by the controller in compliance with the above algorithm, the following normalized values of intensity for the radiators are obtained: $u=\left[\begin{array}{llll}0 & 0.6015 & 1.0000 & 0.2564\end{array}\right]$ (Table 2).

The result obtained is relevant to the contamination pattern as it is located closest to three of the ultrasonic radiators. In the next stage, the intensity of each radiator is set with the amplification factor $u$. The result of the second cleaning cycle is shown in Fig. 9. The necessity to continue cleaning is determined by the module of the vector of signal deviations between the cycles. To complete cleaning, four cycles are required, besides the initial one. Fig. 10 depicts the reduction of the deviation vector module.

\section{CONCLUSIONS}

New methods of ultrasonic cleaning considering the space distribution of the process are developed. The described technology is based on the 3D IT2 fuzzy logic controller, which possesses a structure similar to the traditional one considering spatial and interval characteristics of the membership function. The membership functions considering the space impact of the input parameters are determined. A rule base that remains $2 \mathrm{D}$ and does not depend on the number of sensors and outputs is designed. Assessment of cleaning is based on the difference between the ultrasonic reflections of the latest measurements and the number of dispersed deviations of the latest signal. The functioning algorithm of the described controller determines the amplification factor for each output normalized within $[0,1]$. As some values are $<1$, there is power savings of $41.17 \%$ for the ultrasonic cleaning process modeled herein.

\section{REFERENCES}

1. Duran F., Teke M. (2018), Design and implementation of an intelligent ultrasonic cleaning device, Intelligent Automation and Soft Computing, 441-450.

2. Karnik N., Mendel, J. (2001), Centroid of a type-2 fuzzy set, Inform.Sci., 132, 195-220.

3. Li H., Zhang X., Li, S. (2007), A Three-Dimensional Fuzzy Control Methodology for a Class of Distributed Parameter Systems, IEEE Transactions on Fuzzy Systems, 15(3), 470-481.

4. Mamdani E. (1974), Application of fuzzy logic algorithms for control of simple dynamic plant, Proceedings of the Institution of Electrical Engineers, 121(12), 1585-1588.

5. Mendel J. M., John R. I., Liu, F. (2006), Interval Type-2 Fuzzy Logic Systems Made Simple, IEEE Transactions on Fuzzy Systems, 14(6), 808-821.

6. Morkun V., Morkun N., Tron V. (2015d), Model synthesis of nonlinear nonstationary dynamical systems in concentrating production using Volterra kernel transformation, Metallurgical and Mining Industry, 7(10), 6-9.

7. Morkun V., Morkun N., Pikilnyak A. (2014a), Modeling of ultrasonic waves propagation in inhomogeneous medium using fibered spaces method (k-space). Metallurgical and Mining Industry, 6(2), 43-48.

8. Morkun V., Morkun N., Pikilnyak A. (2014b), The adaptive control for intensity of ultrasonic influence on iron ore pulp, Metallurgical and Mining Industry, 6(6), 8-11.

9. Morkun V., Morkun N., Tron V. (2015a), Distributed closed-loop control formation for technological line of iron ore raw materials beneficiation, Metallurgical and Mining Industry, 7(7),16-19.

10. Morkun V., Morkun N., Tron V. (2015b), Distributed control of ore beneficiation interrelated processes under parametric uncertainty, Metallurgical and Mining Industry, 7(8), 18-21.

11. Morkun V., Morkun N., Tron, V. (2015c), Identification of control systems for ore-processing industry aggregates based on nonparametric kernel estimators, Metallurgical and Mining Industry, 7(1), 14-17.

12. Nigmetzyanov R. I., Kazantsev V. F., Prikhod'ko V. M., Sundukov S. K., Fatyukhin D. S. (2019), Improvement in Ultrasound Liquid Machining by Activating Cavitational Clusters, ISSN 1068-798X, Russian Engineering Research, 8, 699-702.

13. Porkuian O., Morkun V., Morkun N. (2020), Measurement of the ferromagnetic component content in the ore suspension solid phase, Ultrasonics, 105, 106103.

14. Porkuian O., Morkun V., Morkun N., Serdyuk O. (2019), Predictive Control of the Iron Ore Beneficiation Process Based on the Hammerstein Hybrid Model, Acta Mechanica et Automatica, 13(4), 262-270.

15. Rahim A., Bardoshadi H. and Sarrafi S. (2011), Design and Manufacture an Ultrasonic Dispersion System, Sensors and Transducers Journal, 126(3), 52-63.

16. Roohia R., Abedib E., Hashemi S. M. B., Marszałek K., Lorenzo J. M., Barbae F. (2019). Ultrasound-assisted bleaching: Mathematical and 3D computational fluid, Innovative Food Science and Emerging Technologies, 55, 66-79.

17. Tangsopha W., Thongsri J., Busayaporn W. (2017). Simulation of ultrasonic cleaning and ways to improve the efficiency. 5th International Electrical Engineering Congress, 8-10. 
24. Zhang X., Fu Z.-Q., Li S.-Y., Zou T., Wang B. (2017), A time/space separation based 3D fuzzy modeling approach for nonlinear spatially distributed systems, International Journal of Automation and Computing, 15, 1-14.

The authors express their sincere gratitude to Kryvyi Rih National University for support in conducting this research.

Volodymyr Morkun: (D) https://orcid.org/0000-0003-1506-9759

Olha Kravchenko: (D) https://orcid.org/0000-0003-0667-2695 